ERRATUM

doi:10.1038/nature14605

\title{
Erratum: A strong ultraviolet pulse from a newborn type Ia supernova
}

Yi Cao, S. R. Kulkarni, D. Andrew Howell, Avishay Gal-Yam, Mansi M. Kasliwal, Stefano Valenti, J. Johansson, R. Amanullah, A. Goobar, J. Sollerman, F. Taddia, Assaf Horesh, Ilan Sagiv, S. Bradley Cenko, Peter E. Nugent, Iair Arcavi, Jason Surace, P. R.Woźniak, Daniela I. Moody, Umaa D. Rebbapragada, Brian D. Bue \& Neil Gehrels

Nature 521, 328-331 (2015); doi:10.1038/nature14440

In this Letter, the superscript in the ultraviolet luminosity was listed incorrectly as ' -41 ' rather than ' 41 ' in the last sentence of the second paragraph from the bottom in the left column of page 1. It should have read $L_{\mathrm{UV}} \approx 3 \times 10^{41} \mathrm{erg} \mathrm{s}^{-1}$. This has been corrected online. 